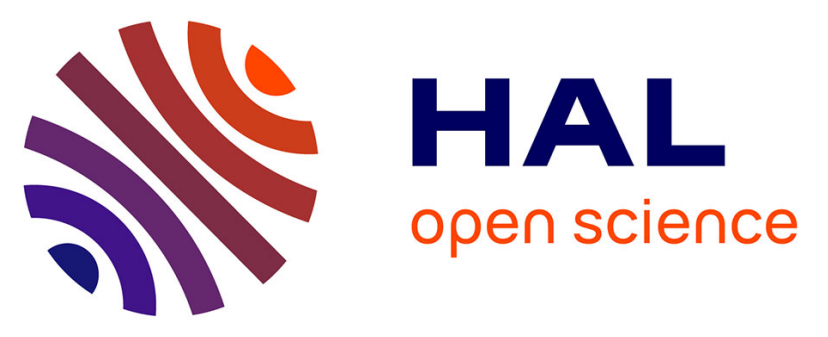

\title{
Group multiple lags consensus of fractional-order nonlinear leader-following multi-agent systems via adaptive control
}

Yunlong Zhang, Guoguang Wen, Zhaoxia Peng, Yongguang Yu, Ahmed Rahmani

\section{To cite this version:}

Yunlong Zhang, Guoguang Wen, Zhaoxia Peng, Yongguang Yu, Ahmed Rahmani. Group multiple lags consensus of fractional-order nonlinear leader-following multi-agent systems via adaptive control. Transactions of the Institute of Measurement and Control, 2018, 41 (5), pp.1313-1322. 10.1177/0142331218777570 . hal-02117332

\section{HAL Id: hal-02117332 \\ https://hal.science/hal-02117332}

Submitted on 2 May 2019

HAL is a multi-disciplinary open access archive for the deposit and dissemination of scientific research documents, whether they are published or not. The documents may come from teaching and research institutions in France or abroad, or from public or private research centers.
L'archive ouverte pluridisciplinaire HAL, est destinée au dépôt et à la diffusion de documents scientifiques de niveau recherche, publiés ou non, émanant des établissements d'enseignement et de recherche français ou étrangers, des laboratoires publics ou privés. 


\title{
Group multiple lags consensus of fractional-order nonlinear leader-following multi-agent systems via adaptive control
}

Transactions of the Institute of Measurement and Control $1-10$

(C) The Author(s) 2018

Reprints and permissions: sagepub.co.uk/journalsPermissions.nav DOI: 10.1 I 77/0 I 4233।218777570 journals.sagepub.com/home/tim @SAGE

\author{
Yunlong Zhang', Guoguang Wen' $\mathbb{D}$, Zhaoxia Peng', \\ Yongguang $\mathrm{Yu}^{1}$ and Ahmed Rahmani ${ }^{3}$
}

\begin{abstract}
In this paper, group multiple lags consensus of fractional-order leader-following multi-agent systems with nonlinear dynamics are investigated, in which two kinds of lag consensus are considered. One is said to be outergroup lag consensus, which means that different group leaders reach lag consensus. The other one is called innergroup lag consensus, that is to say, the followers will reach lag consensus with their own group leader. Based on MittagLeffler stability for fractional-order systems, algebraic graph theory, a class of novel control protocols is designed and the corresponding sufficient conditions are derived to guarantee the achievement of group multiple lags consensus. Furthermore, considering parametric uncertainties, an adaptive control technology is employed to solve the group multiple lags consensus for fractional order multi-agent systems, and the corresponding adaptive control protocols and sufficient conditions are proposed. Finally, numerical simulations are given to demonstrate the effectiveness of the obtained results.
\end{abstract}

\section{Keywords}

Multi-agent systems, lag consensus, adaptive control, fractional-order, Mittag-Leffler stability

\section{Introduction}

It is well-known that the distributed coordination control of multi-agent systems (MASs) have been paid more and more attention from various disciplines, such as applied mathematics, physics, computer sciences and control theory. There is no doubt that it is partly own to its broad applications, for example, unmanned air vehicles (Reynolds, 1987), formation control (Dong and Hu, 2016, 2017; Fax and Murray, 2004; Olfati-Saber and Murray, 2002; Peng et al., 2013, 2016), multiple mobile robots (Chu et al., 2017; Consolini et al., 2008), attitude control (Hu et al., 2017, 2018a,b), flocking and tracking (Olfati-Saber, 2006; Porfiri et al., 2007; Su et al., 2009), etc. Within these applications, one object is to hope all agents reach an agreement on common qualities (position, velocity, phases and attitudes) by communicating with their local neighbours, which is called a consensus problem.

As a fundamental topic in distributed coordination control, consensus problems have received a great deal of attention, and have obtained a lot of interesting results (Cheng et al., 2016a; Xing and Deng, 2017). The previous publications on consensus problems mainly focus on MASs with firstorder linear dynamics (Liu and Liu, 2015; Olfati-Saber and Murray, 2004; Ren and Beard, 2005; Xie and Wang, 2007). Later, many researchers put their vision on second-order dynamics (Mei et al., 2013; Ren and Atkins, 2007; Song et al., 2017; Wen et al., 2016b; Xu et al., 2014; Yu et al., 2013, 2017).
The all articles of first-order and second-order MASs mentioned mainly concern the complete consensus, i.e. the destination of controller design is to drive all the agents achieve a common state. For instance, Liu et al. (2017) investigated the consensus of second-order multi-agent systems by using pulse-modulated intermittent control. However, in many cases, agents may be divided into multiple subgroups due to different environments or tasks, and in each subgroup can reach different consistent state, this is so-called group consensus or cluster consensus. Recently, the topic of group consensus has been extensively studied in many aspects (Gao et al., 2017; Liu and Liu, 2015; Wen et al., 2016c; Xia et al., 2016). In Xia et al. (2016), the authors considered the group consensus of MASs with communication delays under fixed and switching topologies. Miao and Ma (2015) proposed group consensus protocols for discrete-time and continuous-time

\footnotetext{
'Department of Mathematics, Beijing Jiaotong University, Beijing, China ${ }^{2}$ School of Transportation Science and Engineering, Beihang University, Beijing, China

${ }^{3}$ LAGIS UMR 8219 CNRS, Ecole Centrale de Lille, Villeneuve d'Ascq, France
}

\section{Corresponding author:}

Guoguang Wen, Department of Mathematics, Beijing Jiaotong University, Beijing, China.

Email: guoguang.wen@bjtu.edu.cn 
MASs with nonlinear input constrains. By using frequencydomain analysis method and matrix theory, the dynamics group consensus problem of heterogeneous MASs with time delay was investigated in Wen et al. (2016c). In Liu and Liu (2015), the authors investigated the adaptive group consensus of networked Euler-Lagrange systems. Meanwhile, some corresponding necessary and sufficient conditions for solving group consensus problem are established.

It is interesting to note that most of existing research results about consensus or group consensus problems of MASs are integer-order dynamic models (Cheng et al., 2016b; Wen et al., 2016a, 2017a,b). For example, in Liu et al. (2012) an impulsive consensus algorithm was proposed for secondorder continuous-time multi-agent networks with switching topology. In fact, integer-order systems are the special cases of the fractional-order systems, and many phenomenon are difficult to explain in the framework of integer-order dynamics, examples include heat transfer process (Gabano and Poinot, 2011), chemotaxis behaviours and food seeking of microbes (Cohen et al., 2001; Kozlovsky et al., 1999). Besides, fractional-order calculus offer an excellent tool for the description of memory and hereditary properties of various substance and processes, which can describe the systems more precise than the integer-order models. Therefore, it is very meaningful to deal with the consensus problem of fractional-order systems. In the past few decades, the fractional-order MASs have gained considerable research attention (Bai et al., 2017). In Wang et al. (2014), the authors studied the consensus about linear and nonlinear fractionalorder MASs with bounded input time delay and gave some sufficient conditions. By using adaptive pinning control method, $\mathrm{Yu}$ et al. (2015) discussed the leader-following consensus problem of fractional-order MAS and obtained some corresponding criteria based on matrix inequalities.

It is important to point out that most existing research results relative to fractional-order MASs are concerned without time delay. In reality, time delay is unavoidable to exist in many situations. For example, in the consensus of migrating geese or locust population migration, all agents in the same group almost reach the place at the same time, but the different groups arrive at the place in different times. That is, not all agents arrive at the same place simultaneously, but the arrival time of the agents may be different. In addition, proper time delays between different vehicles in the way can keep the road safe and orderly. Otherwise, congestion often occurs. As is known to all, time delay can cause oscillations or in stabilities. Thus, it is extremely important and necessary to investigate fractional-order MAS with time delay. To the best of our knowledge, only a few articles consider the time delay issue in consensus problem of fractional-order MASs. Moreover, the group multiple lags consensus of fractional-order nonlinear MASs of leader-following fractional-order MASs have not been addressed before.

Motivated by the above discussions, in this paper, we consider group multiple lags consensus of fractional-order nonlinear leader-following MASs via adaptive control. The contribution of this paper can be summarized as the following aspects. Firstly, two kinds of lag consensus are defined. One is called as outergroup lag consensus, which means that different group leaders reach lag consensus. The other one is called as innergroup lag consensus, which means the followers reach lag consensus with their own group leaders. Only the two kinds of lag consensus are reached can the group multiple lag consensus be realised. Secondly, to achieve group multiple lags consensus, a class of distributed control protocols is designed and the corresponding sufficient conditions are obtained based on Fractional-order Lyapunov direct method, algebraic graph theory. Thirdly, considering parametric uncertainties that may exist in the leader or follower dynamics, a class of adaptive control protocols for the fractional-order MASs is employed. Then the corresponding sufficient conditions are obtained. Finally, numerical simulations are provided to illustrate the effectiveness of the theoretical results obtained in this paper. Compared with existing works, this paper has the following advantages. In contrast to integer-order models (Reynolds, 1987), formation control (Dong and Hu, 2016, 2017; Fax and Murray, 2004; OlfatiSaber and Murray, 2002; Peng et al., 2013, 2016), this paper considers fractional-order models, which offer an excellent tool for the description of memory and hereditary properties of various substance and processes, as well as describe the systems more precise than the integer-order models. In contrast to the existing literatures (Ma et al., 2015; Wang et al., 2014) that time delays are only between leader and followers or only among leaders, this paper considers two kinds of time delays not only between leader and followers, but also among leaders, which is more considerate and practical.

Notations: In this paper, $I_{N}$ denotes the identity matrix with dimension N. $R^{m \times n}$ denote the set of all $m \times n$ real matrices, $\operatorname{diag}\{\cdots\}$ stands for a block-diagonal matrix. The superscript ' $T$ ' denotes matrix transposition. Notations $\|\cdot\|$ and $\otimes$ denote the Euclidean norm and the Kronecker product respectively. $\lambda_{i}(A)$ is the $i$ th eigenvalue of matrix $A . \lambda_{\max }(A)$ and $\lambda_{\min }(A)$ are the maximum and minimum eigenvalues of $A$, respectively.

\section{Preliminaries and problem description}

\section{Graph theory}

Consider a directed simple graph denoted by $\mathcal{G}=(\mathcal{V}, \mathcal{E}, A)$, where $\mathcal{V}=\left\{v_{1}, v_{2}, \ldots, v_{N}\right\}$ represents the nonempty set of nodes, $\mathcal{E} \subseteq \mathcal{V} \times \mathcal{V}$ is the set of edges. Edge $e_{i j}=\left(v_{i}, v_{j}\right) \in \mathcal{E}$ in a directed graph means that agent $v_{j}$ can receive information from agent $v_{i} . A=\left[a_{i j}\right]_{N \times N}$ denotes the weighted adjacency matrix with nonnegative entries, $a_{i j}>0$ if $e_{j i} \in \mathcal{E}$ and $a_{i j}=0$ if $e_{j i} \notin \mathcal{E}$. Let $D=\operatorname{diag}\left\{d_{1}, d_{2}, \ldots, d_{N}\right\}$ be the $N \times N$ diagonal matrix, where $d_{i}=\operatorname{deg}_{\text {in }}(i)=\sum_{j=1, j \neq i}^{N} a_{i j}$, The Laplacian matrix $L=\left[l_{i j}\right]_{N \times N}$ of graph $\mathcal{G}$ is $L=D-A$.

Then, one has

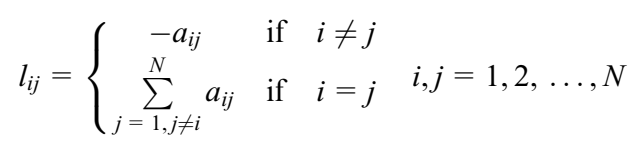

Suppose the $N$ follower agents can be partitioned into $n$ groups $\mathcal{V}_{1}=\left\{v_{1}, v_{2}, \ldots, v_{m_{1}}\right\}, \ldots, \mathcal{V}_{i}=\left\{v_{m_{1}+m_{2}+\ldots+m_{i-1}+1}\right.$, 
$\left.\ldots, \quad v_{m_{1}}+m_{2}+\ldots+m_{i-1}+m_{i}\right\}, \ldots, \quad \mathcal{V}_{n}=\left\{v_{m_{1}}+m_{2}+\ldots+m_{n-1}+1\right.$, $\left.\ldots, v_{N}\right\}$. Let $\hat{i}$ represents the subscript of the group that the $i$ th agent belongs, that is $v_{i} \in \mathcal{V}_{\hat{i}}$. Let $V=\{1,2, \ldots, N\}, \Omega=\{1,2, \ldots, n\}$ denote the subscript set of the followers and leaders, respectively. It is obvious that the followers of the $j$ th leader are in $V_{j}, j \in \Omega$.

\section{Caputo fractional derivative}

In this subsection, the Caputo fractional-order derivative is introduced, which will be used in later section. The Caputo fractional-order derivative of a function $x(t)$ is defined as follows.

Definition 1. (Li et al., 2009)

$$
{ }_{t_{0}} D_{t}^{\alpha} x(t)=\frac{1}{\Gamma(n-\alpha)} \int_{t_{0}}^{t} \frac{x^{(n)}(\tau)}{(t-\tau)^{\alpha+1-n}} d \tau
$$

where $\Gamma(\cdot)$ denotes the Gamma function and $n$ is a positive integer such that $n-1<\alpha<n$.

Property 1. When $C$ is any constant, ${ }_{t} D_{t}^{\alpha} C=0$ holds.

Property 2. For constants $\mu$ and $\nu$, the linearity of Caputo fractional-order derivative is described by

$$
{ }_{t_{0}} D_{t}^{\alpha}(\mu f(t)+\nu g(t))=\mu_{t_{0}} D_{t}^{\alpha} f(t)+\nu_{t_{0}} D_{t}^{\alpha} g(t)
$$

\section{Mittag-Leffler function}

In the following the definition of Mittag-Leffler function is given to deal with the solutions of fractional-order differential equations.

Definition 2. (Li et al., 2009) The Mittag-Leffler function with two parameters is defined as

$$
E_{\alpha, \beta}(z)=\sum_{k=0}^{\infty} \frac{z^{k}}{\Gamma(\alpha k+\beta)}
$$

where $\alpha>0, \beta>0$ and $z \in C$. When $\beta=1$, its one-parameter form is shown as

$$
E_{\alpha}(z)=\sum_{k=0}^{\infty} \frac{z^{k}}{\Gamma(\alpha k+1)}=E_{\alpha, 1}(z)
$$

In particular, $E_{1,1}(z)=e^{z}$.

Definition 3. (Li et al., 2009) The fractional nonautonomous system ${ }_{t_{0}} D_{t}^{\alpha} x(t)=f(t, x)$ with initial condition $x\left(t_{0}\right)$ is said to be Mittag-Leffler stable if the following relationship holds

$$
\|x(t)\| \leq\left[m\left(x\left(t_{0}\right)\right) E_{\alpha}\left(-\lambda\left(t-t_{0}\right)^{\alpha}\right)\right]^{b}
$$

where $x \in R^{n}, \lambda>0, b>0, m(0)=0, m(x) \geq 0$ and $m(x)$ satisfies locally Lipschitz condition on $x$.

Lemma 3.1. (Li et al., 2009) For $t_{0}=0$, the fractional-order system ${ }_{t_{0}} D_{t}^{\alpha} x(t)=f(t, x)$ with initial condition $x\left(t_{0}\right)$ is Mittag-Leffler stable at the equilibrium point $\bar{x}=0$ if there exists a continuously differentiable function $V(t, x(t))$ satisfies

$$
\begin{array}{r}
\alpha_{1}\|x\|^{a} \leq V(t, x(t)) \leq \alpha_{2}\|x\|^{a b} \\
{ }_{0} D_{t}^{\alpha} V(t, x(t)) \leq-\alpha_{3}\|x\|^{a b}
\end{array}
$$

where $V(t, x(t)):[0, \infty) \times D \rightarrow R$ satisfies locally Lipschitz condition on $x ; D \subset R^{n}$ is a domain containing the origin; $t \geq 0$, $\alpha \in(0,1), \alpha_{1}, \alpha_{2}, \alpha_{3}, a$ and $b$ are arbitrary positive constants. If the assumptions hold globally on $R^{n}$, then $\bar{x}=0$ is globally Mittag-Leffler stable.

Lemma 3.2. (Duarte-Mermoud et al., 2015) For any time instant $\geq t_{0}$, the following relationship holds

$$
\frac{1}{2} t_{0} D_{t}^{\alpha} x^{T}(t) P x(t) \leq x^{T}(t) P_{t_{0}} D_{t}^{\alpha} x(t), \forall \alpha \in(0,1]
$$

where $x(t) \in R^{n}$ is a vector of differentiable functions and $P \in R^{n \times n}$ is a constant, square, symmetric, positive definite matrix. Specially, when the matrix $P$ denotes identity matrix $I$, the conclude holds.

\section{Problem description}

Let's consider a fractional-order nonlinear MAS with $N$ followers and $n$ leaders. The dynamics of each follower can be expressed as

$$
D^{\alpha} x_{i}(t)=f\left(x_{i}(t)\right)+\sum_{j \neq i} a_{i j}\left(x_{j}(t)-x_{i}(t)\right)+u_{i}(t), i \in V
$$

where $x_{i}(t) \in R$ and $f\left(x_{i}(t)\right) \in R$ denote the position state and nonlinear intrinsic dynamic of the $i$ th agent, $u_{i}(t) \in R$ denotes the control input. $D^{\alpha}$ denotes the operator of Caputo fractional derivatives. By applying the definition of the Laplacian matrix, the formula (6) can be rewritten as follows

$$
D^{\alpha} x_{i}(t)=f\left(x_{i}(t)\right)-\sum_{j \in V} l_{i j} x_{j}(t)+u_{i}(t), i \in V
$$

or

$$
D^{\alpha} x_{i}(t)=f\left(x_{i}(t)\right)-\sum_{j \in V_{\hat{i}}} l_{i j} x_{j}(t)-\sum_{j \notin V_{\hat{i}}} l_{i j} x_{j}(t)+u_{i}(t), i \in V
$$

Next, the leader's dynamics can be described as

$$
D^{\alpha} s_{j}(t)=f\left(s_{j}(t)\right)-k_{j}\left[s_{j}(t)-s_{1}\left(t-\tau_{j}\right)\right], j=1,2, \ldots, n
$$

where, $s_{j}(t)$ denotes the $j$ th leader's position state, $k_{j}>0$, the time delay $\tau_{1}=0$ and $\tau_{j}>0$ for $j \in\{2, \ldots, n\}$. It is obvious that $s_{1}(t)=f\left(s_{1}(t)\right)$. Note from (9) that the first leader is the leader of the first group, meanwhile it is also the leader of other leaders. In (6) and (9), $f(\cdot)$ is the nonlinear inherent dynamics of agent, and satisfies the following Assumption 1.

Assumption 1. For any vectors,$y \in R^{m}$, there exists a constant $\gamma>0$ satisfies $(x-y)^{T}(f(x)-f(y)) \leq \gamma(x-y)^{T}(x-y)$.

Definition 4. The fractional-order nonlinear MASs (9) reach outergroup lag consensus, if for any initial conditions, 
$\lim _{t \rightarrow \infty} \sum_{j=2}^{n}\left\|e_{j}^{\tau}(t)\right\|=0$, where $e_{j}^{\tau}(t)=s_{j}(t)-s_{1}\left(t-\tau_{j}\right)$, where $\tau_{j}$ are the time delays among different group leaders.

Definition 5. The fractional-order nonlinear leader-following MASs (6) and (9) reach innergroup lag consensus, if for any initial conditions, $\lim _{t \rightarrow \infty} \sum_{i=1}^{N}\left\|\eta_{i}(t)\right\|=0$, where $\eta_{i}(t)=x_{i}(t)-$ $s_{\hat{i}}\left(t-p_{\hat{i}}\right), p_{\hat{i}}$ are the time delays that between followers and their own group leaders.

Definition 6. The fractional-order nonlinear leader-following MASs with (6) and (9) reach group multiple lags consensus(GMLC), if for any initial conditions, $\lim _{t \rightarrow \infty} \sum_{i=1}^{N}\left\|\eta_{i}(t)\right\|=0$ and $\lim _{t \rightarrow \infty} \sum_{j=2}^{n}\left\|e_{j}^{\tau}(t)\right\|=0$, where $\eta_{i}(t)=x_{i}(t)$ $-s_{\hat{i}}\left(t-p_{\hat{i}}\right)$, and $e_{j}^{\tau}(t)=s_{j}(t)-s_{1}\left(t-\tau_{j}\right)$, where $p_{\hat{i}}$ are the time delays that between followers and their own group leaders, $\tau_{j}$ are the time delays among different group leaders.

Remark 1. In this article, all agents are supposed to be in one-dimensional space, i.e. $x_{i}(t), s_{j}(t), f\left(x_{i}(t)\right), u_{i}(t) \in R$, in this way, it is convenient for us to prove the main result. However, we can extend our result in $n$-dimensional space by employing the Kronecker product. In other words, the results obtained in this paper for $x_{i}(t), s_{j}(t), f\left(x_{i}(t)\right), u_{i}(t) \in R^{m}$ are still valid.

\section{Main results}

\section{Group multiple lags consensus of fractional-order nonlinear leader-following MASs}

In order to reach group multiple lags consensus, we propose the following control inputs for the system (6)

$$
\begin{aligned}
u_{i}(t)= & -k_{\hat{i}}\left(s_{\hat{i}}\left(t-p_{\hat{i}}\right)-s_{1}\left(t-p_{\hat{i}}-\tau_{\hat{i}}\right)\right) \\
& -\sigma_{i}\left(x_{i}(t)-s_{\hat{i}}\left(t-p_{\hat{i}}\right)\right) \\
& +\sum_{j \notin V_{\hat{i}}} l_{i j}\left(x_{j}(t)-x_{i}(t)\right), i \in V
\end{aligned}
$$

where $p_{\hat{i}}$ are the time delays that between leaders and followers, $\tau_{\hat{i}}$ are the time delays among different group leaders, $k_{\hat{i}}>0$ and $\sigma_{i}>0$.

Theorem 6.1. Under Assumption 1, the fractional-order MASs (6) and (9) with the control protocol (10) reach group multiple lags consensus, that is to say, $\lim _{t \rightarrow \infty} \sum_{i=1}^{N}\left\|\eta_{i}(t)\right\|=0$ and $\lim _{t \rightarrow \infty} \sum_{j=2}^{n}\left\|e_{j}^{\tau}(t)\right\|=0$, if the matrix $H=\gamma I_{N}-\sigma-\frac{1}{2}\left(M+M^{T}\right)$ is negative definite and $\min _{j \in \Omega, j \neq 1} k_{j}>\gamma$, where $M=\left(m_{i j}\right)_{N \times N}$, $m_{i j}=l_{i j}$ for $j \in V_{\hat{i}}$ and $i \neq j, m_{i j}=0$ for $j \notin V_{\hat{i}}, m_{i i}=-\sum_{j \neq i} m_{i j}$, $\sigma=\operatorname{diag}\left\{\sigma_{1}, \sigma_{2}, \ldots, \sigma_{N}\right\}$.

Proof: We prove the theorem by two steps. The first step is to prove that $\lim _{t \rightarrow \infty} \sum_{i=1}^{N}\left\|\eta_{i}(t)\right\|=0$, and the second step is to prove $\lim _{t \rightarrow \infty} \sum_{j=2}^{n}\left\|e_{j}^{\tau}(t)\right\|=0$.
The first step: Denote $\eta_{i}(t)=x_{i}(t)-s_{\hat{i}}\left(t-p_{\hat{i}}\right), \eta(t)=\left(\eta_{1}(t)\right.$, $\left.\eta_{2}(t), \ldots, \eta_{N}(t)\right)^{T}, \quad f(x(t))=\left(f\left(x_{1}(t)\right), f\left(x_{2}(t)\right), \ldots, \quad f\left(x_{N}(t)\right)\right)^{T}$, and $f(s(t-p))=\left(f\left(s_{1}\left(t-p_{1}\right)\right), f\left(s_{2}\left(t-p_{2}\right)\right), \ldots, f\left(s_{N}\left(t-p_{N}\right)\right)\right)^{T}$. By (8), (9), (10) and the definition of $M$, we can obtain

$$
D^{\alpha} \eta_{i}(t)=\left(f\left(x_{i}(t)\right)-f\left(s_{\hat{i}}\left(t-p_{\hat{i}}\right)\right)\right)-\sum_{j \in V_{\hat{i}}} m_{i j} \eta_{j}(t)-\sigma_{i} \eta_{i}(t)
$$

Furthermore, equation (11) can be written in the matrix form as follows

$$
\begin{aligned}
D^{\alpha} \eta(t) & =(f(x(t))-f(s(t-p)))-M \eta(t)-\sigma \eta(t) \\
& =f(x(t))-f(s(t-p))-(M+\sigma) \eta(t)
\end{aligned}
$$

Construct the following Lyapunov function candidate

$$
V_{1}(t)=\frac{1}{2} \eta^{T}(t) \eta(t)
$$

Combining with Lemma 3.2, take the time derivative of (13) as

$$
\begin{aligned}
D^{\alpha} V_{1}(t) & \leq \eta^{T}(t) D^{\alpha} \eta(t) \\
& \leq \eta^{T}(t)(f(x(t))-f(s(t-p))-(M+\sigma) \eta(t)) \\
& \leq \eta^{T}(t)(\gamma I-M-\sigma) \eta(t) \\
& =\eta^{T}(t)\left(\gamma I-\frac{1}{2}\left(M^{T}+M\right)-\sigma\right) \eta(t) \\
& =\eta^{T}(t) H \eta(t) \leq \lambda_{\max }(H)\|\eta(t)\|
\end{aligned}
$$

Since $H<0$, it follows from Lemma 3.1 that the fractionalorder MAS (6) and (9) satisfy satisfy $\lim _{t \rightarrow \infty}\|\eta(t)\|=0$, i.e. $\lim _{t \rightarrow \infty} \sum_{i=1}^{N}\left\|\eta_{i}(t)\right\|=0$.

The second step: By (9), we can obtain

$$
\begin{aligned}
& D^{\alpha} e_{j}^{\tau}(t)=\left(f\left(s_{j}(t)\right)-f\left(s_{1}\left(t-\tau_{j}\right)\right)\right)-k_{j} e_{j}^{\tau}(t), \\
& j=2,3, \ldots, n
\end{aligned}
$$

Let $e^{\tau}(t)=\left(\left(e_{2}^{\tau}(t)\right), \ldots,\left(e_{n}^{\tau}(t)\right)\right)^{T}, K=\operatorname{diag}\left\{k_{2}, \ldots, k_{n}\right\}$, $f(s(t))=\left(f\left(s_{2}(t)\right), f\left(s_{3}(t)\right), \ldots, f\left(s_{n}(t)\right)\right)^{T}$, and $f\left(s_{1}(t-\tau)\right)=$ $\left(f\left(s_{1}\left(t-\tau_{2}\right)\right), f\left(s_{1}\left(t-\tau_{3}\right)\right), \ldots, f\left(s_{1}\left(t-\tau_{n}\right)\right)\right)^{T}$. Then

$$
D^{\alpha} e^{\tau}(t)=\left(f(s(t))-f\left(s_{1}(t-\tau)\right)\right)-K e^{\tau}(t)
$$

Construct the following Lyapunov function

$$
V_{2}(t)=\frac{1}{2}\left(e^{\tau}(t)\right)^{T} e^{\tau}(t)
$$

Combining with Lemma 3.2, calculate the derivative of $V_{2}(t)$ as

$$
\begin{aligned}
D^{\alpha} V_{2}(t) & \leq\left(e^{\tau}(t)\right)^{T} D^{\alpha} e^{\tau}(t) \\
& \leq\left(e^{\tau}(t)\right)^{T}\left(\left(f(s(t))-f\left(s_{1}(t-\tau)\right)\right)-K e^{\tau}(t)\right) \\
& \leq\left(e^{\tau}(t)\right)^{T}\left(\gamma I_{n-1}-K\right) e^{\tau}(t) \\
& \leq \lambda_{\max }\left(\gamma I_{n-1}-K\right)\left\|e^{\tau}(t)\right\|
\end{aligned}
$$


Since $\min _{j \in \Omega, j \neq 1} k_{j}>\gamma$, it follows that $\gamma I_{n-1}-K$ is a negative definite matrix. Furthermore, according to Lemma 3.1, one has $\lim _{t \rightarrow \infty}\left\|e^{\tau}(t)\right\|=0$, that is to say, $\lim _{t \rightarrow \infty} \sum_{j=2}^{n}\left\|e_{j}^{\tau}(t)\right\|=0$.

Through the above two steps of proof, we can say that the group multiple lags consensus of the fractional-order MASs with (6) and (9) is achieved under the control inputs (10).

Remark 2. In the process of proof, we split it into two parts. In the first step, we prove that the agents in the same group can reach innergroup lag consensus, in other words, all followers can track their own leaders with time delay. The second part prove that the group leaders in different groups can reach outergroup lag consensus, it is to say, all group leaders can track the first group leader with time delay. Only the above two parts are finished, can the group multiple lags consensus be completed.

Corollary 6.2. Under Assumption 1, the fractional-order MASs (6) and (9) with the following control protocol

$$
\begin{aligned}
u_{i}(t)=- & k_{\hat{i}}\left(s_{\hat{i}}(t)-s_{1}\left(t-\tau_{\hat{i}}\right)\right)-\sigma_{i}\left(x_{i}(t)-s_{\hat{i}}(t)\right) \\
& +\sum_{j \notin V_{\hat{i}}} l_{i j}\left(x_{j}(t)-x_{i}(t)\right), i \in V
\end{aligned}
$$

reach outergroup lag consensus, that is to say, $\lim _{t \rightarrow \infty} \sum_{i=1}^{N}\left\|x_{i}(t)-s_{\hat{i}}(t)\right\|=0 \quad$ and $\quad \lim _{t \rightarrow \infty} \sum_{j=2}^{n}\left\|e_{j}^{\tau}(t)\right\|=0$, if $\min _{j \in \Omega, j \neq 1} k_{j}>\gamma$ and $H=\gamma I_{N}-\sigma-\frac{1}{2}\left(M+M^{T}\right)$ is negative definite, where $M=\left(m_{i j}\right)_{N \times N}, m_{i j}=l_{i j}$ for $j \in V_{\hat{i}}$ and $i \neq j$, $m_{i j}=0$ for $j \subsetneq V_{\hat{i}}, m_{i i}=-\sum_{j \neq i} m_{i j}$.

Proof: The proof is similar to Theorem 6.1, therefore is omitted here.

\section{Group multiple lags consensus in the fractional order MASs via adaptive control}

In this subsection, considering the parameter uncertainty, we first employ adaptive control to solve the group multiple lags consensus for fractional order MASs. The dynamics of the leaders are given by

$$
D^{\alpha} s_{j}(t)=f\left(s_{j}(t)\right)-k_{j}(t)\left(s_{j}(t)-s_{1}\left(t-\tau_{j}\right)\right), j=1,2, \ldots, n
$$

where the time delay $\tau_{1}=0, \tau_{j}>0$ for $j \in\{2, \ldots, n\}$. Obviously, $D^{\alpha} s_{1}(t)=f\left(s_{1}(t)\right)$. The gain function $k_{j}(t)$ in $(20)$ is continuous, and its derivative under Caputo definitions is given by

$$
D^{\alpha} k_{j}(t)=b\left(s_{j}(t)-s_{1}\left(t-\tau_{j}\right)\right)^{T}\left(s_{j}(t)-s_{1}\left(t-\tau_{j}\right)\right)
$$

where $b$ is a positive constant. Based on the definition of $e_{j}^{\tau}(t)$, the equation (21) can be rewritten as

$$
D^{\alpha} k_{j}(t)=b\left(e_{j}^{\tau}(t)\right)^{T} e_{j}^{\tau}(t)
$$

In order to solve the group multiple lags consensus of system (6) and (20), the following adaptive control protocol is given as

$$
\begin{aligned}
u_{i}(t)= & -k_{\hat{i}}(t)\left(s_{\hat{i}}\left(t-p_{\hat{i}}\right)-s_{1}\left(t-p_{\hat{i}}-\tau_{\hat{i}}\right)\right) \\
& -\sigma_{i}(t)\left(x_{i}(t)-s_{\hat{i}}\left(t-p_{\hat{i}}\right)\right) \\
& +\sum_{j \notin V_{\hat{i}}} l_{i j}\left(x_{j}(t)-x_{i}(t)\right), i \in V
\end{aligned}
$$

where $p_{\hat{i}}$ are the time delays that between followers and their own group leaders, $\tau_{j}$ are the time delays among different group leaders, the derivative of $k_{i}(t)$ is same as (22), and the derivative of $\sigma_{i}(t)$ is given as

$$
D^{\alpha} \sigma_{i}(t)=d\left(x_{i}(t)-s_{\hat{i}}\left(t-p_{\hat{i}}\right)\right)^{T}\left(x_{i}(t)-s_{\hat{i}}\left(t-p_{\hat{i}}\right)\right)
$$

with $d$ being a positive constant. Based on the definition of $\eta_{i}(t)$, the equation (24) can be rewritten as

$$
D^{\alpha} \sigma_{i}(t)=d \eta_{i}^{T}(t) \eta_{i}(t)
$$

By (20), we can obtain

$$
\begin{aligned}
D^{\alpha} s_{\hat{i}}\left(t-p_{\hat{i}}\right)=- & k_{\hat{i}}\left(s_{\hat{i}}\left(t-p_{\hat{i}}\right)-s_{1}\left(t-p_{\hat{i}}-\tau_{\hat{i}}\right)\right) \\
& +f\left(s_{\hat{i}}\left(t-p_{\hat{i}}\right)\right), \hat{i}=1,2, \ldots, n
\end{aligned}
$$

It then follows from (6) and (26), we have

$$
D^{\alpha} \eta_{i}(t)=f\left(x_{i}(t)\right)-f\left(s_{\hat{i}}\left(t-p_{\hat{i}}\right)\right)-\sigma_{i}(t) \eta_{i}(t)-\sum_{j \in V_{\hat{i}}} m_{i j} \eta_{j}(t)
$$

Rewrite (27) in matrix form as follows

$$
D^{\alpha} \eta(t)=f(x(t))-f(s(t-p))-\sigma(t) \eta(t)-M \eta(t)
$$

Theorem 6.3. Under Assumption 1, the fractional-order system (6) and (20) with the control protocol (23) reach delay consensus, that is to say, $\lim _{t \rightarrow \infty} \sum_{i=1}^{N}\left\|\eta_{i}(t)\right\|=0 \quad$ and $\lim _{t \rightarrow \infty} \sum_{j=2}^{n}\left\|e_{j}^{\tau}(t)\right\|=0$, if $\tilde{k}>\gamma$ and $Q=\gamma I_{N}-\tilde{\sigma}-\frac{1}{2}\left(M+M^{T}\right)$ is negative definite, where $M=\left(m_{i j}\right)_{N \times N}, m_{i j}=l_{i j}$ for $j \in V_{\hat{i}}$ and $i \neq j, m_{i j}=0$ for $j \subsetneq V_{\hat{i}}, m_{i i}=-\sum_{j \neq i} m_{i j}, \tilde{k}, \tilde{\sigma}$ is an undetermined sufficiently large positive constant.

Proof: We prove the theorem still by two steps. First, we prove that $\lim _{t \rightarrow \infty} \sum_{i=1}^{N}\left\|\eta_{i}(t)\right\|=0$. Construct the Lyapunov function as follows

$$
V_{3}(t)=\frac{1}{2} \eta^{T}(t) \eta(t)+\frac{1}{2 d}(\sigma(t)-\tilde{\sigma})^{2}
$$

where $\sigma(t)=\operatorname{diag}\left\{\sigma_{1}(t), \sigma_{2}(t), \ldots, \sigma_{N}(t)\right\}$ and $\tilde{\sigma}$ is an undetermined sufficiently large positive constant. Combining with Lemma 3.2, taking derivative of $V_{3}(t)$ yields 


$$
\begin{aligned}
D^{\alpha} V_{3}(t) & \leq \eta^{T}(t) D^{\alpha} \eta(t)+\frac{1}{d}(\sigma(t)-\tilde{\sigma}) D^{\alpha} \sigma(t) \\
& \leq \eta^{T}(t) f\left(x(t)-f(s(t-p))-\eta^{T}(t) M \eta(t)\right. \\
& -\sigma(t) \eta^{T}(t) I_{N} \eta(t)+\frac{1}{d}(\sigma(t)-\tilde{\sigma}) D^{\alpha} \sigma(t) \\
& \leq \eta^{T}(t)\left(\gamma I_{N}-M-\tilde{\sigma} I_{N}\right) \eta(t) \\
& =\eta^{T}(t)\left(\gamma I_{N}-\frac{1}{2}\left(M^{T}+M\right)-\tilde{\sigma} I_{N}\right) \eta(t) \\
& =\eta^{T}(t) Q \eta(t) \leq \lambda_{\max }(Q)\|\eta(t)\|
\end{aligned}
$$

Since $Q$ is a negative definite matrix, it then follows from Lemma 3.1 that the solutions of the fractional-order MAS satisfy $\lim _{t \rightarrow \infty}\|\eta(t)\|=0$. It implies that $\lim _{t \rightarrow \infty} \sum_{i=1}^{N}\left\|\eta_{i}(t)\right\|=0$.

Next, we prove $\lim _{t \rightarrow \infty} \sum_{j=2}^{n}\left\|e_{j}^{\tau}(t)\right\|=0$. Consider the following Lyapunov function

$$
V_{4}(t)=\frac{1}{2}\left(e^{\tau}(t)\right)^{T} \times e^{\tau}(t)+\frac{1}{2 b}(K(t)-\tilde{k})^{2}
$$

where $K(t)=\operatorname{diag}\left\{k_{2}(t), \ldots, k_{n}(t)\right\}$ and $\tilde{k}$ is an undetermined sufficiently large positive constant. Combining with Lemma 3.2 , take the derivative of $V_{4}(t)$ as

$$
\begin{aligned}
D^{\alpha} V_{4}(t) & \leq \frac{1}{2}\left(e^{\tau}(t)\right)^{T}\left(f(s(t))-f\left(s_{1}(t-\tau)\right)\right) \\
& +\frac{1}{2}\left(e^{\tau}(t)\right)^{T}\left(f(s(t))-f\left(s_{1}(t-\tau)\right)\right) \\
& -K(t)\left(e^{\tau}(t)\right)^{T} e^{\tau}(t)+(K(t)-\tilde{k})\left(e^{\tau}(t)\right)^{T} e^{\tau}(t) \\
& \left.=e^{\tau}(t)\right)^{T}\left(f(s(t))-f\left(s_{1}(t-\tau)\right)\right)-\tilde{k} I_{n-1}\left(e^{\tau}(t)\right)^{T} e^{\tau}(t) \\
& \leq\left(e^{\tau}(t)\right)^{T} \times\left(\gamma I_{n-1}-\tilde{k} I_{n-1}\right) e^{\tau}(t) \\
& \leq \lambda_{\max }\left((\gamma-\tilde{k}) I_{n-1}\right)\|e(t)\|
\end{aligned}
$$

Since $\tilde{k}>\gamma$, it follows that $(\gamma-\tilde{k}) I_{n-1}$ is a negative definite matrix. Furthermore, according to Lemma 3.1, we can get $\lim _{t \rightarrow \infty}\left\|e^{\tau}(t)\right\|=0$, that is to say, $\lim _{t \rightarrow \infty} \sum_{j=2}^{n}\left\|e_{j}^{\tau}(t)\right\|=0$.

Combining the above two steps, the proof is completed.

Corollary 6.4. Under Assumption 1, the fractional-order system (6) and (20) with the following control protocol

$$
\begin{aligned}
u_{i}(t)=- & k_{\hat{i}}(t)\left(s_{\hat{i}}(t)-s_{1}\left(t-\tau_{\hat{i}}\right)\right)-\sigma_{i}(t)\left(x_{i}(t)-s_{\hat{i}}(t)\right) \\
& +\sum_{j \notin V_{\hat{i}}} l_{i j}\left(x_{j}(t)-x_{i}(t)\right), i \in V
\end{aligned}
$$

with (21) and(24) reach outergroup lag consensus via adaptive control, that is to say, $\lim _{t \rightarrow \infty} \sum_{i=1}^{N}\left\|x_{i}(t)-s_{\hat{i}}(t)\right\|=0$ and $\lim _{t \rightarrow \infty} \sum_{j=2}^{n}\left\|e_{j}^{\tau}(t)\right\|=0$, if $\tilde{k}>\gamma$ and $Q=\gamma I_{N}-\tilde{\sigma}-\frac{1}{2}\left(M+M^{T}\right)$ is negative definite, where $M=\left(m_{i j}\right)_{N \times N}, m_{i j}=l_{i j}$ for $j \in V_{\hat{i}}$ and $i \neq j, m_{i j}=0$ for $j \subsetneq V_{\hat{i}}, m_{i i}=-\sum_{j \neq i} m_{i j}$.

Proof: The proof is similar to Theorem 6.3, therefore is omitted here.

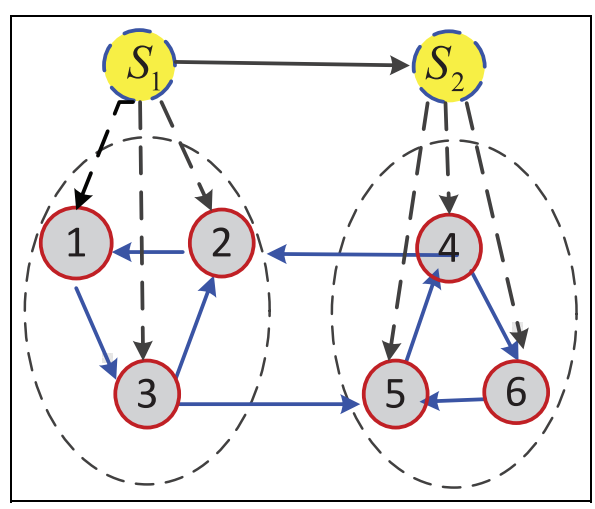

Figure I. The communication topology of the MAS, where $s_{j}$ denotes the $j$ th leader, and $i$ denotes the $i$ th follower, $j=I, 2 ; i=1,2, \ldots, 6$.

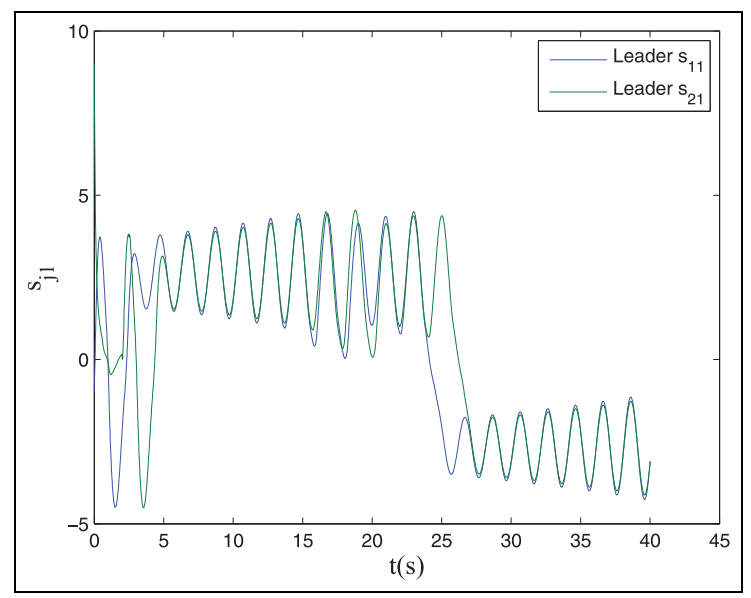

Figure 2. The first component $s_{j 1}$ of the jth leader's state, where $j=I, 2$.

\section{Simulation results}

In this section, in order to validate our theoretical results, the following simulation example is presented. We consider a MASs consists of two leaders (indexed by $S_{1}$ and $S_{2}$ ) and six followers (indexed by 1 to 6 ), whose communication topology is shown in Figure 1. From Figure 1, we can see that there are two subgroups, $S_{1}$ and $S_{2}$ are the leaders of the two subgroups, and $S_{1}$ is the leader of $S_{2}$. In addition, we can obtain that

$$
\begin{aligned}
A & =\left(\begin{array}{cccccc}
0 & 1 & 0 & 0 & 0 & 0 \\
0 & 0 & 1 & 1 & 0 & 0 \\
1 & 0 & 0 & 0 & 0 & 0 \\
0 & 0 & 0 & 0 & 1 & 0 \\
0 & 0 & 1 & 0 & 0 & 1 \\
0 & 0 & 0 & 1 & 0 & 0
\end{array}\right), \\
L & =\left(\begin{array}{ccccccc}
1 & -1 & 0 & 0 & 0 & 0 \\
0 & 2 & -1 & -1 & 0 & 0 \\
-1 & 0 & 1 & 0 & 0 & 0 \\
0 & 0 & 0 & 1 & -1 & 0 \\
0 & 0 & -1 & 0 & 2 & -1 \\
0 & 0 & 0 & -1 & 0 & 1
\end{array}\right)
\end{aligned}
$$




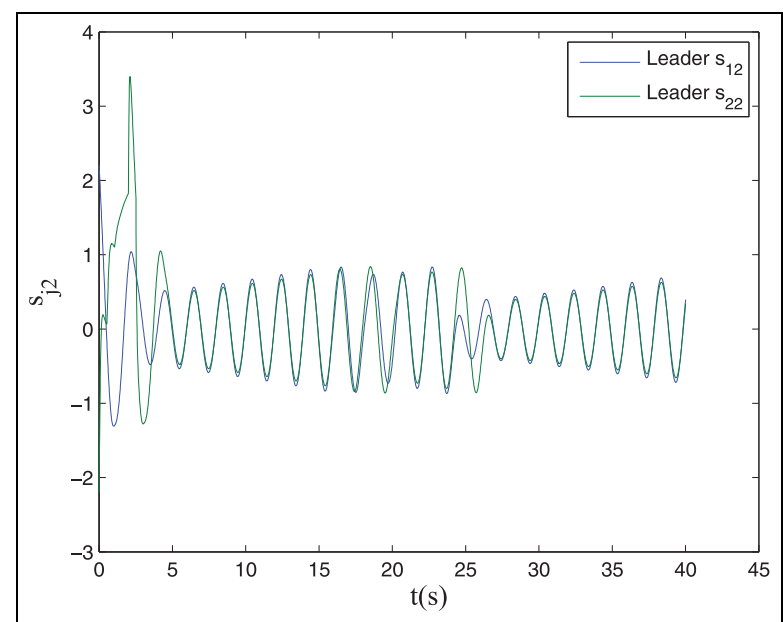

Figure 3. The second component $s_{j 2}$ of the jth leader's state, where $j=I, 2$.

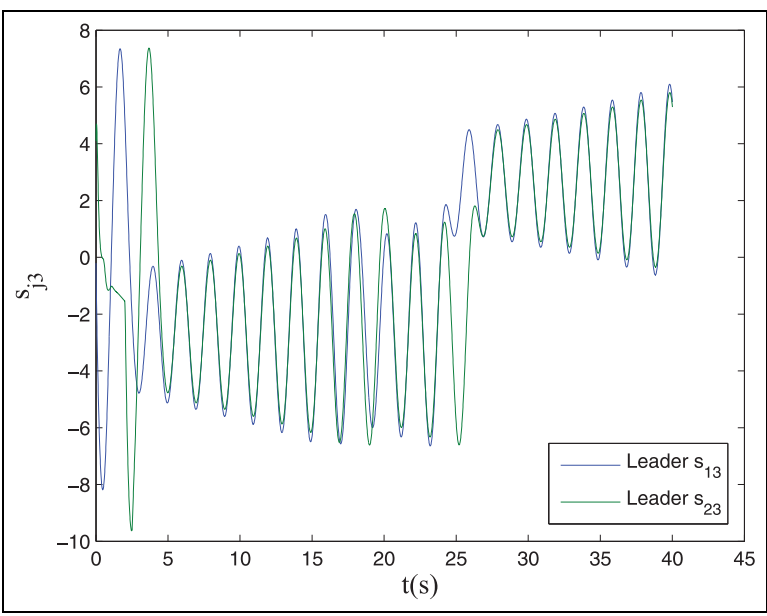

Figure 4. The third component $s_{j 3}$ of the jth leader's state, where $j=1,2$.

and

$$
M=\left(\begin{array}{cccccc}
1 & -1 & 0 & 0 & 0 & 0 \\
0 & 1 & -1 & 0 & 0 & 0 \\
-1 & 0 & 1 & 0 & 0 & 0 \\
0 & 0 & 0 & 1 & -1 & 0 \\
0 & 0 & 0 & 0 & 1 & -1 \\
0 & 0 & 0 & -1 & 0 & 1
\end{array}\right)
$$

Without loss of generality, in this section we just verify the results obtained in Theorem 6.3 and Corollary 6.4 because of the limitation of space. Let $f(\cdot)$ in (6) and (9) be the Chua network (Ma et al., 2015), which is described by

$$
f=\left(\begin{array}{l}
9 x_{i 2}(t)-\frac{18}{7} x_{i 1}(t)+\frac{24}{7}\left(\left|x_{i 1}(t)+1\right|-\left|x_{i 1}(t)-1\right|\right) \\
x_{i 1}(t)-x_{i 2}(t)+x_{i 3}(t) \\
-\frac{100}{7} x_{i 2}(t)
\end{array}\right)
$$

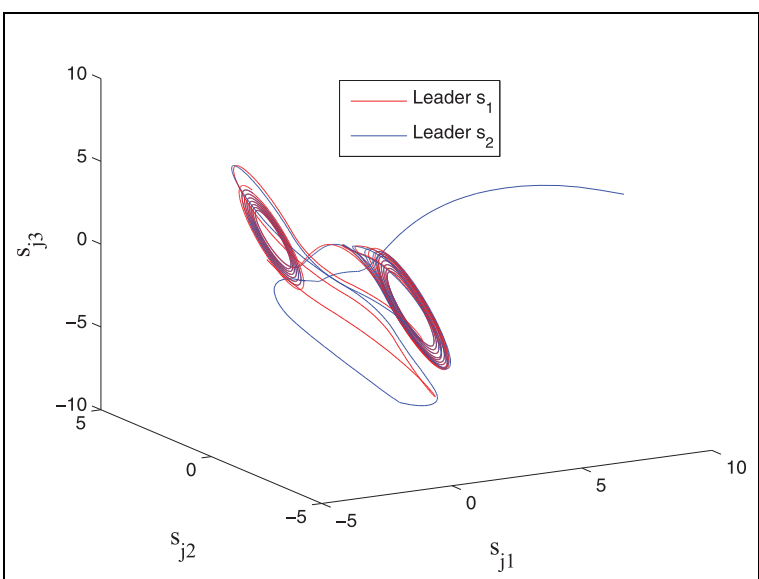

Figure 5. The phase diagram of the leaders $s_{1}$ and $s_{2}$.

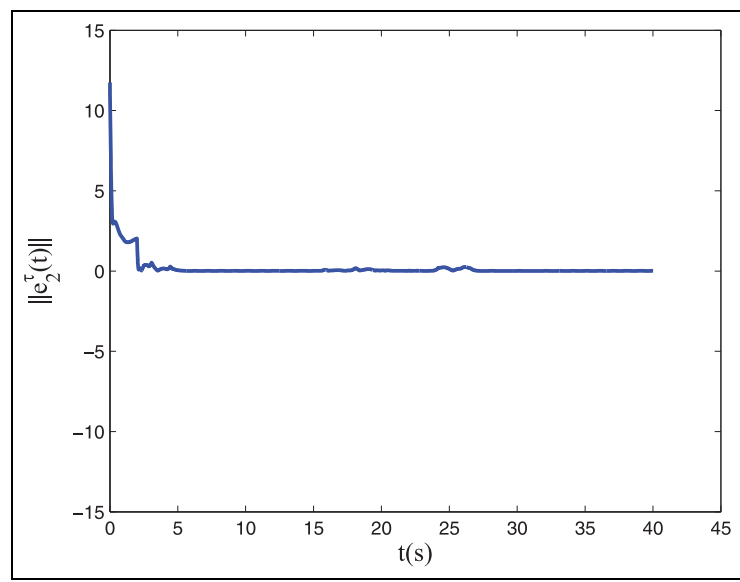

Figure 6. The state error $\left\|e_{2}^{\tau}(t)\right\|=\left\|s_{2}(t)-s_{1}(t-2)\right\|$.

where $x_{i}(t)=\left(x_{i 1}(t), x_{i 2}(t), x_{i 3}(t)\right)^{T}$ represents the state variable of the $i$ th agent. It is easy to calculate that $\gamma$ is equal to 9.1, which satisfies the Assumption 1. Choose $\alpha=0.97$, $\sigma_{i}(0)=0.5, \quad k_{2}(0)=0.5, \quad \tau_{2}=2 \quad$ and $\quad p_{\hat{i}}=0.5$, where $i=1, \ldots, 6$.

Figures 2 to 4 show the three components $s_{j 1}, s_{j 2}$ and $s_{j 3}$ of the $j$ th leader's state, respectively, where $j=1,2$. Figure 5 shows the phase diagram of the leaders $S_{1}$ and $S_{2}$. Figure 6 shows the state error $\left\|e_{2}^{\tau}(t)\right\|=\left\|s_{2}(t)-s_{1}(t-2)\right\|$. From Figure 2 to Figure 6, we can see that the leader $S_{1}$ and leader $S_{2}$ reach outergroup lag consensus. Figure 7 shows the first components $x_{i 1}$ and $s_{11}$ of the agents states in the first group with $p_{\hat{i}}=0.5$,where $i=1,2,3$. Figure 8 shows the first components $x_{i 1}$ and $s_{21}$ of the agents' states in the second group with $p_{i}=0.5$,where $i=4,5,6$. Figure 9 shows the state error $\left\|\eta_{i}(t)\right\|=\left\|x_{i}(t)-s_{\hat{i}}(t-0.5)\right\|$, where $i=1, \ldots, 6$. Figure 10 shows the time evolution of $k_{2}(t)$ and $\sigma_{i}(t)$, where $i=1, \ldots, 6$. From Figures 7 to 9, agents 1, 2 and 3 reach innergroup lag consensus with leader $S_{1}$, and agents 4,5 and 6 reach innergroup lag consensus with leader $S_{2}$. From the above simulation results, the group multiple lags consensus has achieved, the Theorem 6.3 is verified. In the following we 


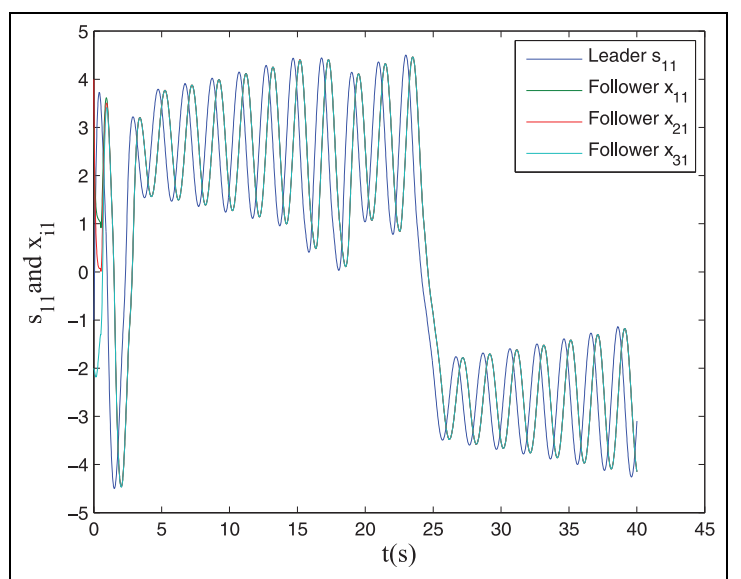

Figure 7. The first components $x_{i 1}$ and $s_{||}$of the agents' states in the first group with $p_{i}=0.5$, where $i=1,2,3$.

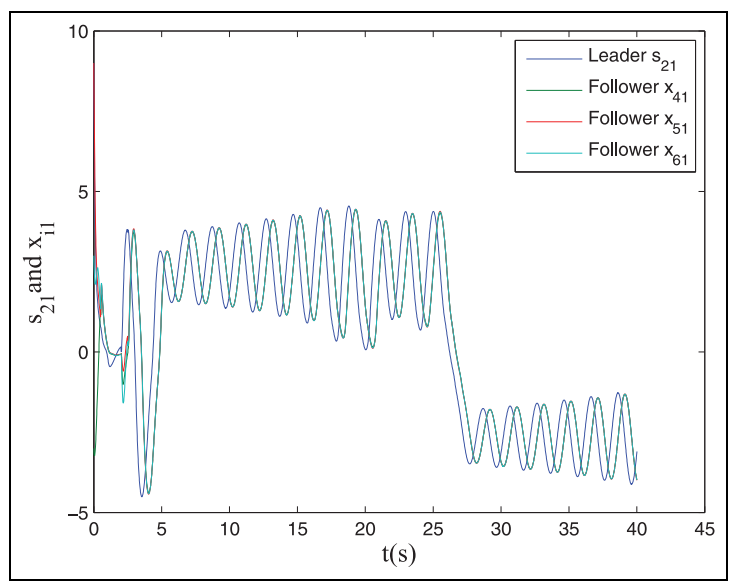

Figure 8. The first components $x_{i 1}$ and $s_{21}$ of the agents' states in the second group with $p_{i}=0.5$, where $i=4,5,6$.

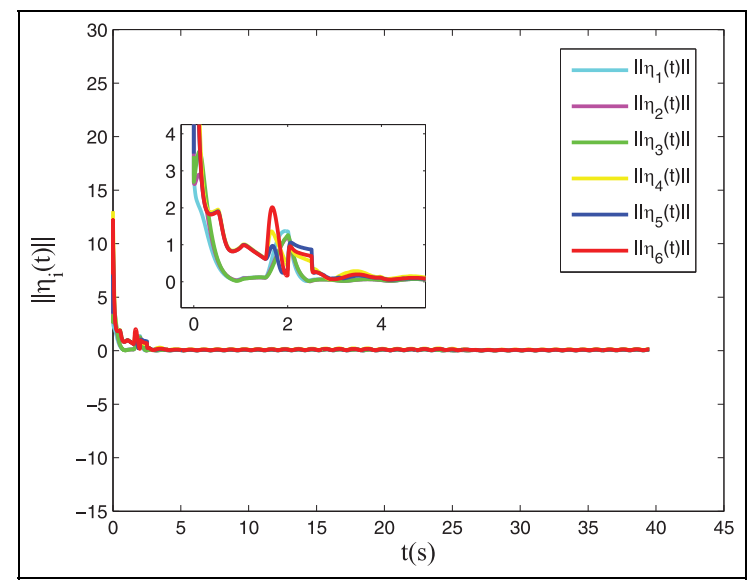

Figure 9. The state error $\left\|\eta_{i}(t)\right\|=\left\|x_{i}(t)-s_{i}(t-0.5)\right\|$, where $i=1, \ldots, 6$.

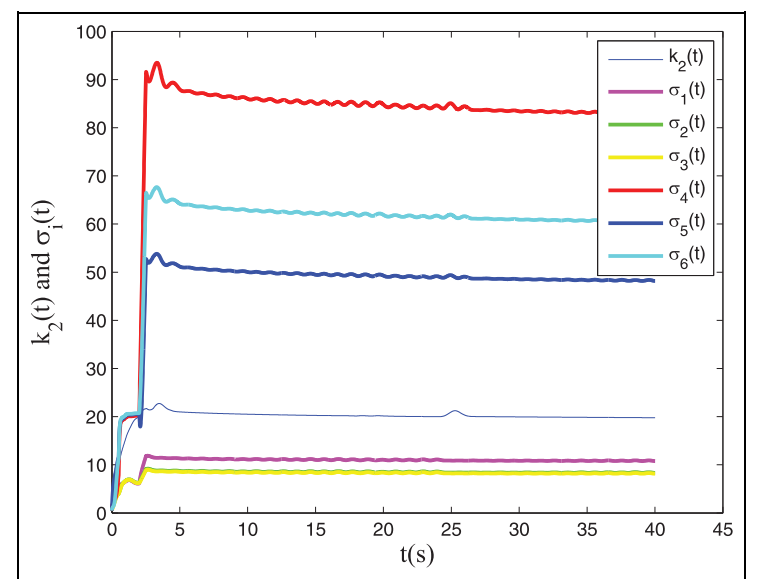

Figure 10. The time evolution of $k_{2}(t)$ and $\sigma_{i}(t)$, where $i=1, \ldots, 6$.

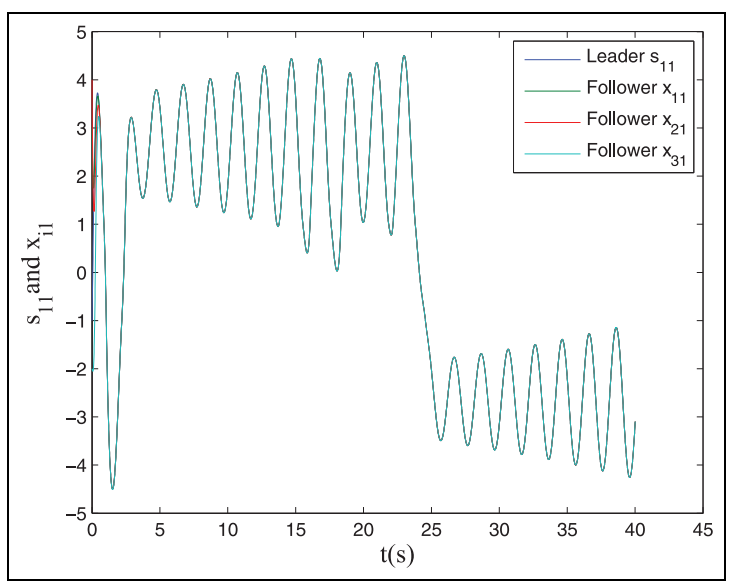

Figure II. The first components $x_{i 1}$ and $s_{\mid 1}$ of the agents' states in the first group with $p_{i}=0$, where $i=1,2,3$.

consider the case that $p_{\hat{i}}=0$. Figure 11 shows the first components $x_{i 1}$ and $s_{11}$ of the agents' states in the first group with $p_{\hat{i}}=0$, where $i=1,2,3$. Figure 12 shows the first components $x_{i 1}$ and $s_{21}$ of the agents' states in the second group with $p_{\hat{i}}=0$, where $i=4,5,6$. Figure 13 shows the state error $\left\|e_{i}(t)\right\|=\left\|x_{i}(t)-s_{\hat{i}}(t)\right\|$, where $i=1, \ldots, 6$. we can see from Figures 11 to 13 that the outergroup lag consensus has been archived, that is to say, Corollary 6.4 has been verified.

\section{Conclusions}

This paper has investigated the group multiple lags consensus in fractional-order leader-following MAS with nonlinear dynamics via adaptive control. A class of novel control protocols and adaptive control protocols have been designed respectively. The corresponding sufficient conditions have been derived based on Fractional-order Lyapunov direct method, algebraic graph theory. Our future works will mainly 


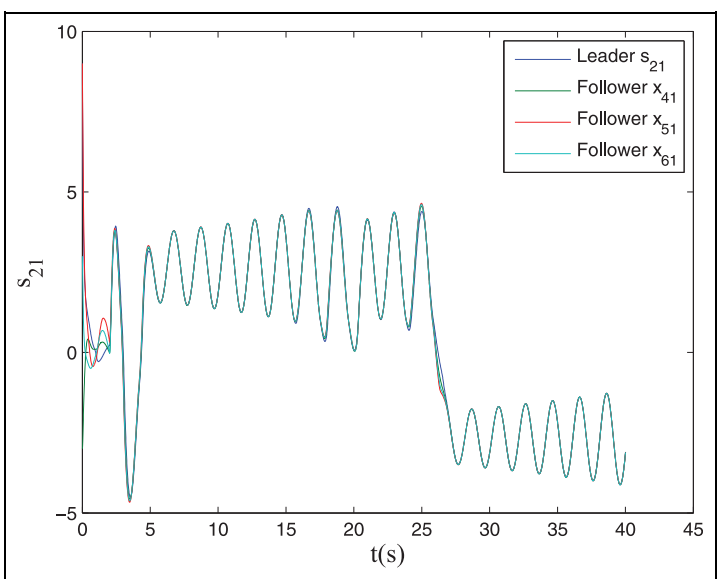

Figure 12. The first components $x_{i 1}$ and $s_{21}$ of the agents' states in the second group with $p_{i}=0$,where $i=4,5,6$.

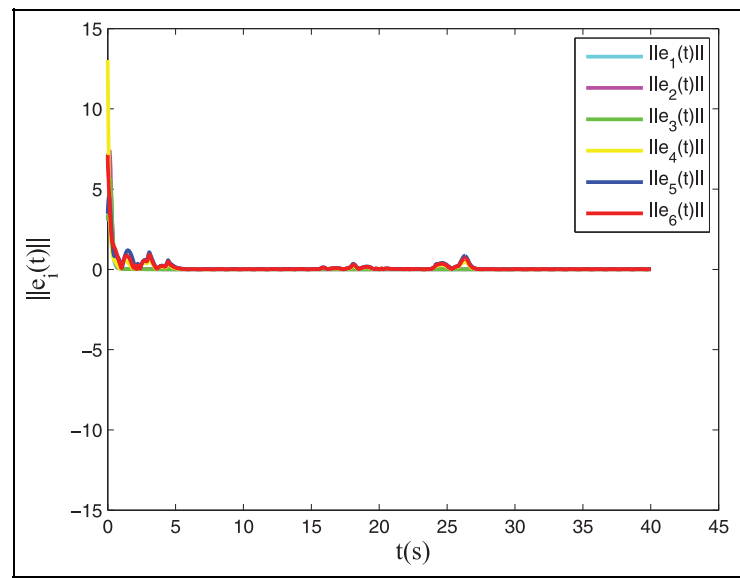

Figure 13. The state error $\left\|e_{i}(t)\right\|=\left\|x_{i}(t)-s_{i}(t)\right\|$, where $i=1, \ldots, 6$.

concentrate on group multiple lags consensus of fractionalorder MAS under switching topologies or with time-varying reference states via pinning control.

\section{Declaration of Conflicting Interests}

The author(s) declared no potential conflicts of interest with respect to the research, authorship, and/or publication of this article.

\section{Funding}

The author(s) disclosed receipt of the following financial support for the research, authorship, and/or publication of this article: This work was supported by the National Natural Science Foundation of China under Grants 61503016 and 61403019, the Fundamental Research Funds for the Central Universities under Grants YWF-15-SYS-JTXY-007, YWF16-BJ-Y-21 and 2017JBM067, and the National Key Research and Development Program of China under Grant 2017 YFB0103202.

\section{ORCID iD}

Guoguang Wen iDhttps://orcid.org/0000-0001-9128-1802

\section{References}

Bai J, Wen G, Rahmani A, et al. (2017) Distributed consensus tracking for the fractional-order multi-agent systems based on the sliding mode control method. Neurocomputing 235: 210-216.

Cheng L, Wang Y, Ren W, et al. (2016a) Containment control of multiagent systems with dynamic leaders based on a $p i^{n}$-type approach. IEEE Transactions on Cybernetics 46(12): 3004-3017.

Cheng L, Wang Y, Ren W, et al. (2016b) On convergence rate of leader-following consensus of linear multi-agent systems with communication noises. IEEE Transactions on Automatic Control 61(11): 3586-3592.

Chu X, Peng Z, Wen G, et al. (2017) Distributed formation tracking of multi-robot systems with nonholonomic constraint via eventtriggered approach. Neurocomputing 275: 121-131.

Cohen I, Golding I, Ron IG, et al. (2001) Biofluiddynamics of lubricating bacteria. Mathematical Methods in the Applied Sciences 24(17-18): 1429-1468.

Consolini L, Morbidi F, Prattichizzo D, et al. (2008) Leader-follower formation control of nonholonomic mobile robots with input constraints. Automatica 44(5): 1343-1349.

Dong X and Hu G (2016) Time-varying formation control for general linear multi-agent systems with switching directed topologies. Automatica 73: 47-55.

Dong X and Hu G (2017) Time-varying formation tracking for linear multiagent systems with multiple leaders. IEEE Transactions on Automatic Control 62(7): 3658-3664.

Duarte-Mermoud MA, Aguila-Camacho N, Gallegos JA, et al. (2015) Using general quadratic Lyapunov functions to prove Lyapunov uniform stability for fractional order systems. Communications in Nonlinear Science and Numerical Simulation 22(1-3): 650-659.

Fax JA and Murray RM (2004) Information flow and cooperative control of vehicle formations. IEEE Transactions on Automatic Control 49(9): 1465-1476.

Gabano JD and Poinot T (2011) Fractional modelling and identification of thermal systems. Signal Processing 91(3): 531-541.

Gao Y, Yu J, Yu M, et al. (2017) Couple-group consensus for secondorder multi-agent systems with the effect of second-order neighbours information. Transactions of the Institute of Measurement and Control 40: 1726-1737.

$\mathrm{Hu}$ Q, Jiang B and Zhang Y (2017) Observer-based output feedback attitude stabilization for spacecraft with finite-time convergence. IEEE Transactions on Control Systems Technology (99): $1-9$.

Hu Q, Shao X and Chen WH (2018a) Robust fault-tolerant tracking control for spacecraft proximity operations using time-varying sliding mode. IEEE Transactions on Aerospace and Electronic Systems 54(1): 2-17.

Hu Q, Shao X and Guo L (2018b) Adaptive fault-tolerant attitude tracking control of spacecraft with prescribed performance. IEEE/ ASME Transactions on Mechatronics 23(1): 331-341.

Kozlovsky Y, Cohen I, Golding I, et al. (1999) Lubricating bacteria model for branching growth of bacterial colonies. Physical Review E: Statistical Physics Plasmas Fluids and Related Interdisciplinary Topics 59(6): 703-705.

Li Y, Chen Y and Podlubny I (2009) Mittagcleffler stability of fractional order nonlinear dynamic systems. Automatica 45(8): 1965-1969.

Liu CL and Liu F (2015) Adaptive consensus algorithms for heterogeneous first-order multi-agent systems with diverse nominal 
velocities. Transactions of the Institute of Measurement and Control 38(11): 125-134.

Liu Z, Guan Z, Shen X, et al. (2012) Consensus of multi-agent networks with aperiodic sampled communication via impulsive algorithms using position-only measurements. IEEE Transactions on Automatic Control 57(10): 2639-2643.

Liu Z, Yu X, Guan Z, et al. (2017) Pulse-modulated intermittent control in consensus of multiagent systems. IEEE Transactions on Systems Man and Cybernetics Systems 47(5): 783-793.

Ma Z, Wang Y and Li X (2015) Cluster-delay consensus in first-order multi-agent systems with nonlinear dynamics. Nonlinear Dynamics 83(3): 1303-1310.

Mei J, Ren W and Ma G (2013) Distributed coordination for secondorder multi-agent systems with nonlinear dynamics using only relative position measurements. Automatica 49(5): 1419-1427.

Miao G and Ma Q (2015) Group consensus of the first-order multiagent systems with nonlinear input constraints. Neurocomputing 161: 113-119.

Olfati-Saber R (2006) Flocking for multi-agent dynamic systems: Algorithms and theory. IEEE Transactions on Automatic Control 51(3): 401-420.

Olfati-Saber R and Murray RM (2002) Distributed cooperative control of multiple vehicle formations using structural potential functions. In: IFAC world congress, volume 15, Barcelona, Spain, 2126 July 2002, pp. 242-248. Laxenburg, Austria: Citeseer.

Olfati-Saber R and Murray RM (2004) Consensus problems in networks of agents with switching topology and time-delays. IEEE Transactions on Automatic Control 49(9): 1520-1533.

Peng Z, Wen G, Rahmani A, et al. (2013) Leader-follower formation control of nonholonomic mobile robots based on a bioinspired neurodynamic based approach. Robotics and Autonomous Systems 61(9): 988-996.

Peng Z, Yang S, Wen G, et al. (2016) Adaptive distributed formation control for multiple nonholonomic wheeled mobile robots. Neurocomputing 173: 1485-1494.

Porfiri M, Roberson DG and Stilwell DJ (2007) Tracking and formation control of multiple autonomous agents: A two-level consensus approach. Automatica 43(8): 1318-1328.

Ren W and Atkins E (2007) Distributed multi-vehicle coordinated control via local information exchange. International Journal of Robust and Nonlinear Control 17(10-11): 1002-1033.

Ren W and Beard RW (2005) Consensus seeking in multiagent systems under dynamically changing interaction topologies. IEEE Transactions on Automatic Control 50(5): 655-661.

Reynolds CW (1987) Flocks, herds and schools: A distributed behavioral model. ACM Siggraph Computer Graphics 21(4): 25-34.

Song Q, Liu F, Wen G, et al. (2017) Distributed position-based consensus of second-order multiagent systems with continuous/ intermittent communication. IEEE Transactions on Cybernetics 47(8): 1860-1871.

Su H, Wang X and Lin Z (2009) Flocking of multi-agents with a virtual leader. IEEE Transactions on Automatic Control 54(2): 293-307.

Wang B, Jian J and Shen Y (2014) Consensus of fractional-order multi-agent systems with input delays and compounded orders. In: Chinese control conference, Nanjing, China, 28-30 July 2014, pp. 1110-1114. Beijing, China.

Wen G, Huang J, Peng Z, et al. (2016a) On pinning group consensus for heterogeneous multi-agent system with input saturation. Neurocomputing 207: 623-629.

Wen G, Yu Y, Peng Z, et al. (2016b) Consensus tracking for secondorder nonlinear multi-agent systems with switching topologies and a time-varying reference state. International Journal of Control 89(10): 2096-2106.

Wen G, Yu Y, Peng Z, et al. (2016c) Dynamical group consensus of heterogenous multi-agent systems with input time delays. Neurocomputing 175: 278-286.

Wen G, Yu W, Li Z, et al. (2017a) Neuro-adaptive consensus tracking of multiagent systems with a high-dimensional leader. IEEE Transactions on Cybernetics 47(7): 1730-1742.

Wen G, Yu W, Xia Y, et al. (2017b) Distributed tracking of nonlinear multiagent systems under directed switching topology: An observer-based protocol. IEEE Transactions on Systems, Man, and Cybernetics: Systems 47(5): 869-881.

Xia H, Huang T, Shao J, et al. (2016) Group consensus of multiagent systems with communication delays. Neurocomputing 171: 1666-1673.

Xie G and Wang L (2007) Consensus control for a class of networks of dynamic agents. International Journal of Robust and Nonlinear Control 17(10-11): 941-959.

Xing M and Deng F (2017) Scaled consensus for multi-agent systems with communication time delays. Transactions of the Institute of Measurement and Control 40(8): 2651-2659.

$\mathrm{Xu} \mathrm{C}$, Zheng Y, Su H, et al. (2014) Necessary and sufficient conditions for distributed containment control of multi-agent systems without velocity measurement. IET Control Theory and Applications 8(16): 1752-1759.

Yu W, Ren W, Zheng G, et al. (2013) Distributed control gains design for consensus in multi-agent systems with second-order nonlinear dynamics. Automatica 49(7): 2107-2115.

$\mathrm{Yu} \mathrm{W}$, Wang H, Cheng F, et al. (2017) Second-order consensus in multiagent systems via distributed sliding mode control. IEEE Transactions on Cybernetics 47(8): 1872-1881.

$\mathrm{Yu} \mathrm{Z}$, Jiang $\mathrm{H}$ and $\mathrm{Hu} \mathrm{C}$ (2015) Leader-following consensus of fractional-order multi-agent systems under fixed topology. Neurocomputing 149: 613-620. 